Article

\title{
Immunomodulatory Effect of Marine Cembrane-Type Diterpenoids on Dendritic Cells
}

\section{Ching-Yen Lin ${ }^{1}$, Mei-Chin Lu ${ }^{2}$, Jui-Hsin Su ${ }^{2}$, Ching-Liang Chu ${ }^{3}$, David Shiuan ${ }^{1}$, Ching-Feng Weng ${ }^{1,2}$, Ping-Jyun Sung ${ }^{1,2}$ and Kao-Jean Huang ${ }^{1} *$}

1 Department of Life Science and Institute of Biotechnology, National Dong Hwa University, Hualien 974, Taiwan; E-Mails: jouyuan22@gmail.com (C.-Y.L.); shiuan@ mail.ndhu.edu.tw (D.S.); cfweng@mail.ndhu.edu.tw (C.-F.W.)

2 Graduate Institute of Marine Biotechnology, National Dong Hwa University, Pingtung 944, Taiwan; E-Mails: jinx6609@nmmba.gov.tw (M.-C.L.); x2219@nmmba.gov.tw (J.-H.S.); pjsung@nmmba.gov.tw (P.-J.S.)

3 Graduate Institute of Immunology, College of Medicine, National Taiwan University, Taipei 100, Taiwan; E-Mail: clchu01@ntu.edu.tw

* Author to whom correspondence should be addressed; E-Mail: kj_huang@mail.ndhu.edu.tw; Tel.: +886-3-863-3675; Fax: +886-3-863-3630.

Received: 26 February 2013; in revised form: 22 March 2013 / Accepted: 27 March 2013 / Published: 22 April 2013

Abstract: Dendritic cells (DCs) are antigen presenting cells, which can present antigens to T-cells and play an important role in linking innate and adaptive immunity. DC maturation can be induced by many stimuli, including pro-inflammatory cytokines and bacterial products, such as lipopolysaccharides (LPS). Here, we examined the immunomodulatory effects of marine cembrane compounds, $(9 E, 13 E)$-5-acetoxy-6-hydroxy-9,13-dimethyl-3methylene-3,3a,4,5,6,7,8,11,12,14a-decahydro- $2 H$-cyclotrideca[b]furan-2-one $(\mathbf{1}),(9 E, 13 E)$ 5-acetoxy-6-acetyl-9, 13-dimethyl-3-methylene-3,3a,4,5,6,7,8,11,12,14a-decahydro-2 $H$-cyclo trideca[b]furan-2-one (2), lobocrassin B (3), (-)14-deoxycrassin (4), cembranolide B (5) and 13-acetoxysarcocrassolide (6) isolated from a soft coral, Lobophytum crassum, on mouse bone marrow-derived dendritic cells (BMDCs). The results revealed that cembrane-type diterpenoids, especially lobocrassin B, effectively inhibited LPS-induced BMDC activation by inhibiting the production of TNF- $\alpha$. Pre-treatment of BMDCs with Lobocrassin B for $1 \mathrm{~h}$ is essential to prohibit the following activation induced by various toll-like receptor (TLR) agonists, such as LPS, zymosan, lipoteichoic acid (LTA) and Pam2CSK4. Inhibition of $\mathrm{NF}-\kappa \mathrm{B}$ nuclear translocation by lobocrassin $\mathrm{B}$, which is a key transcription factor for cytokine production in TLR signaling, was evident as assayed by high-content image 
analysis. Lobocrassin B attenuated DC maturation and endocytosis as the expression levels of MHC class II and the co-stimulatory molecules were downregulated, which may affect the function of DCs to initiate the T-cell responses. Thus, lobocrassin B may have the potential in treatment of immune dysregulated diseases in the future.

Keywords: antigen presentation; bone-marrow derived dendritic cell (BMDC); marine natural compound; tumor necrosis factor- $\alpha$

\section{Introduction}

Marine compounds have emerged as the new era in discovering new therapeutic drugs, including those can regulate the inflammatory responses [1-3]. Recently, chemical investigations on soft coral Lobophytum crassum have led to the isolation and identification of varieties of oxygenated cembrane-type diterpenoids [4-8]. The in vitro assay model for screening compounds with anti-inflammatory activity was conducted in lipopolysaccharide (LPS)-treated RAW 264.7 and assayed for the expression levels of pro-inflammatory proteins, particularly the inducible nitric oxide synthase (iNOS) and cyclooxygenase-2 (COX-2). Some cembranoids have been shown to have anti-inflammatory effects by downregulating the iNOS and COX-2 expression [9-12]. In addition, sinularin, a cembrane-type diterpenoid isolated from Sinularia sp., exerted its bioactivities on anti-inflammatory effect, as well as the analgesic property both in vitro and in vivo. In LPS-stimulated RAW cells, sinularin significantly inhibits iNOS and COX-2 expression and upregulates the production of transforming growth factor- $\beta$ (TGF- $\beta$ ), which may contribute to the suppression of microglial and astrocyte activation in carrageenan-induced tissue inflammatory responses [13].

Dendritic cells (DCs) are the professional antigen-presenting cells, which are important in linking the innate and adaptive immunity [14]. DC maturation can be induced by many stimuli, including pro-inflammatory cytokines and bacterial products, such as LPS. Immature DCs bear the pattern recognition receptors (PRRs) for microbes, such as toll-like receptors (TLRs), possess endocytosis ability to load antigens and then migrate toward the draining lymph nodes, where they mature and present the proteasome-degraded antigens to naive T-cells [15]. Mature DCs stop antigen-loading, but enhance the expression of major histocompatibility complexes (MHC) and accessory molecules, such as CD86, CD80 and CD40, and then produce cytokines and chemokines [16]. The signaling cascade responsible for LPS-stimulated DCs is involved the TLR4 binding lipopolysaccharide (LPS), p38 mitogen-activated protein kinases (MAPKs) activation and Nuclear Factor-kappa B (NF-кB) activation [17]. In general, NF- $\kappa \mathrm{B}$ is retained in cytoplasm and complexes with the inhibitor of $\kappa \mathrm{B}(\mathrm{I}-\kappa \mathrm{B})$ repressor


subsequently degraded by ubiquitin-mediated proteasomal degradation and NF- $\kappa \mathrm{B}$ dissociated from $\mathrm{I}-\kappa \mathrm{B}$ in cytoplasm and then translocated into nucleus to activate the inflammatory cytokine and chemokine genes [18]. Thus, DCs play the role in controlling infectious diseases, as well as cancers [19]. However, DCs also participated in the pathogenesis of several immune dysregulated diseases, including chronic inflammation and autoimmunity [20,21]. Intervention of such disease progression may be achieved by suppression or attenuation of DC activation. 
In this study, we examined the immuno-regulatory effect of cembrane-type diterpenoids on bone marrow-derived dendritic cells (BMDCs). Our results showed that lobocrassin B may be a potent immunosuppressant to inhibit DC maturation and activation, suggesting that lobocrassin B may have therapeutic applications in certain immune dysfunctions.

\section{Results and Discussion}

\subsection{Suppression of TNF- $\alpha$ Expression in BMDCs by Cembrane-Type Diterpenoids}

The cembrane-type metabolites were isolated from soft octocoral, Lobophytum crassum, and the cytotoxic effects of these compounds on BMDCs were assayed by the MTT method. Among them, Compound 6 was more toxic to BMDCs, with the cytotoxicity $\left(\mathrm{CC}_{20}\right)$ about $4.2 \mu \mathrm{M}$. The others were less toxic, as their $\mathrm{CC}_{20}$ were 6-9-fold higher than Compound 6 (Table 1). These compounds were tested for their potency to affect the function of BMDCs by measuring TNF- $\alpha$ production. Compounds 1-6 did not induce TNF- $\alpha$ production in immature BMDCs (data not shown), but lobocrassin B (3) at $\mathrm{CC}_{20}$ concentration had the potency to suppress the TNF- $\alpha$ production from LPS-stimulated BMDCs (Figure 1A). We further tested compounds $\mathbf{4}$ and $\mathbf{5}$, which are structurally similar to lobocrassin B for their activities to suppress the TNF- $\alpha$ production. Compounds $\mathbf{4}$ and $\mathbf{5}$ could suppress TNF- $\alpha$ production, but they caused more cell growth inhibition (60 and 30\%, respectively) at the same concentration $(39 \mu \mathrm{M})$ as used for lobocrassin B (Figure 1B). The direct cell cytotoxicity caused by compound 1-6 was further confirmed by Annexin V-propidium iodide (PI) staining after treatment with individual compounds for $6 \mathrm{~h}$. The direct cytotoxicity mediated by lobocrassin B was 10.7\%, and this was less toxic than $33.5 \%, 41 \%$ and $25 \%$ for compounds 4, 5 and $\mathbf{6}$, respectively (Figure 1C). Hence, lobocrassin B could be a potent suppressor that can inhibit TNF- $\alpha$ production from LPS-activated BMDCs, and compounds structurally similar to lobocrassin B may also exert the same effect, but to a different extent.

\subsection{Blocking of Various TLR Agonists-Mediated TNF- $\alpha$ Production in BMDCs by Lobocrassin B}

As lobocrassin B is effective in blocking TNF- $\alpha$ production in LPS-stimulated BMDCs, we further confirmed the duration for lobocrassin B's effect. Pre-treatment of lobocrassin B on BMDCs as short as $1 \mathrm{~h}$ was enough to inhibit TNF- $\alpha$ production from LPS-stimulated BMDCs (Figure 2A), while post-treatment had no effect on the inhibition of TNF- $\alpha$ production (Figure 2B), indicating that lobocrassin B may act on the upstream of LPS-stimulating signaling. As LPS is a potent activator for BMDCs, once its signaling was initiated, lobocrassin B may not be able to counteract this vast impact.

The capacity of lobocrassin B for inhibiting the LPS-stimulated TNF- $\alpha$ production was evaluated. Lobocrassin B could suppress the TNF- $\alpha$ production of BMDCs when they were stimulated by LPS at as high concentration as $1000 \mathrm{ng} / \mathrm{mL}$ (Figure 2C). In addition, we tested whether lobocrassin B could exert the same inhibitory effect when BMDCs were stimulated by various TLR agonists other than LPS. BMDCs were stimulated by Zymosan (for TLR2), Pam (for TLR2) or LTA (for TLR2), and the suppression of TNF- $\alpha$ production for each was evident, where the inhibitory effects by lobocrassin B for each agonists were comparable to that of LPS, i.e., equal or less than $20 \%$ in TNF- $\alpha$ production. Thus, lobocrassin B could be used as a blocker acting on the early events in TLR-related signaling (Figure 2D). 
Table 1. The cytotoxic effect of marine cembrane-type diterpenoids on murine bone marrow-derived dendritic cells (BMDCs).

\begin{tabular}{|c|c|c|c|}
\hline Compound & Structure & $\begin{array}{c}\text { Molecular } \\
\text { Weight }\end{array}$ & $\mathrm{CC}_{20}(\mu \mathrm{M}) *$ \\
\hline $\begin{array}{c}(9 E, 13 E) \text {-5-Acetoxy-6-hydroxy-9, } \\
\text { 13-dimethyl-3-methylene-3,3a,4,5,6,7,8,11, } \\
\text { 12,14a-decahydro-2H-cyclotrideca } \\
{[b] \text { furan-2-one }(\mathbf{1})}\end{array}$ & & 332.43 & 38 \\
\hline $\begin{array}{c}(9 E, 13 E) \text {-5-Acetoxy-6-acetyl-9, } \\
\text { 13-dimethyl-3-methylene-3,3a,4,5,6,7,8,11, } \\
\text { 12,14a-decahydro-2 } H \text {-cyclotrideca } \\
{[b] \text { furan-2-one (2) }}\end{array}$ & & 374.47 & 33 \\
\hline Lobocrassin B (3) & & 318.45 & 39 \\
\hline$(-) 14-D e o x y c r a s s i n(4)$ & & 318.45 & 26.9 \\
\hline Cembranolide B (5) & & 330.42 & 36 \\
\hline 13-Acetoxysarcocrassolide (6) & & 374.47 & 4.2 \\
\hline
\end{tabular}

* Cell cytotoxicity test in murine BMDCs was determined by MTT assay.

Figure 1. Suppression of TNF- $\alpha$ production in lipopolysaccharides (LPS)-stimulated BMDCs by cembrane-type diterpenoids. (A) Inhibition of TNF- $\alpha$ production in BMDCs by coral cembranolides. BMDCs $\left(2 \times 10^{5} /\right.$ well $)$ in 96-well plates were untreated or treated with $0.1 \mu \mathrm{g} / \mathrm{mL}$ of LPS with individual cembranolides $(\mathbf{1}, \mathbf{2}, \mathbf{3}$ (lobocrassin B) or 6) at a concentration of $\mathrm{CC}_{20}$ for $6 \mathrm{~h}$, and mTNF- $\alpha$ levels in the culture supernatant were measured by ELISA. Cell toxicity evaluated by the MTT method was also included. (B) Dose-effects of lobocrassin B (3) and lobocrassin B-related compounds (4 and 5) on inhibition of TNF- $\alpha$ production in LPS-stimulated BMDCs. BMDCs $\left(2 \times 10^{5} /\right.$ well $)$ were untreated or treated with $0.1 \mu \mathrm{g} / \mathrm{mL}$ of LPS plus indicated concentrations of lobocrassin B (3), 4 and 5 for $6 \mathrm{~h}$, 
and mTNF- $\alpha$ levels in the culture supernatant were measured by ELISA. The relative mTNF- $\alpha$ production (\%) in each group was normalized to the LPS-stimulated control (LPS plus DMOS). Bars represent the relative mTNF- $\alpha$ production, and lines represent the survival rate. The data present as the mean \pm SD. $* * p<0.01 v s$. LPS-stimulated control; *** $p<0.001$ vs. LPS-stimulated control. (C) Direct cytotoxicity of compound 1-6 on LPS-stimulated BMDCs. BMDCs incubated with compound 1-6 at the concentration used in (A) for $6 \mathrm{~h}$ were harvested and stained with PI and Annexin V-fluorescein isothiocyanate (FITC) for flow cytometry. The percentage of apoptotic cells (Annexin $\mathrm{V}^{+}$and Annexin $\mathrm{V}^{+} \mathrm{PI}^{+}$), dead cells $\left(\mathrm{PI}^{+}\right)$and survival cells (Annexin $\left.\mathrm{V}^{-} \mathrm{PI}^{-}\right)$were calculated and plotted.

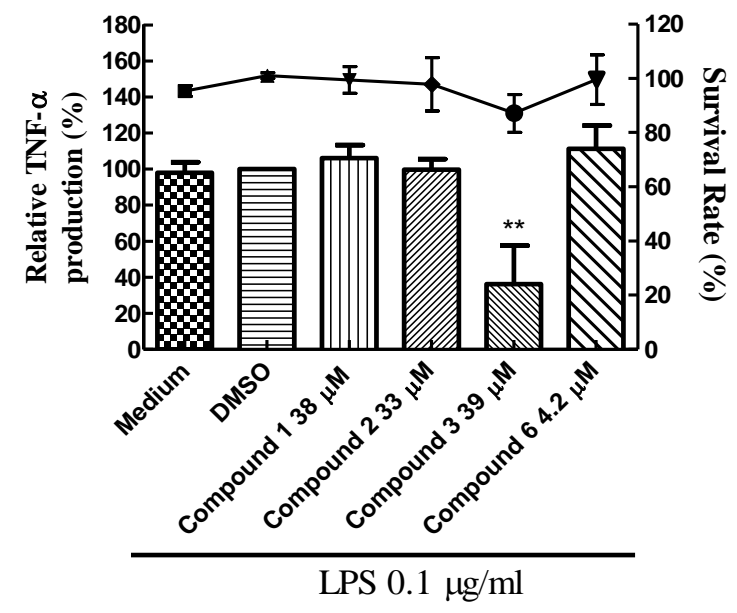

(A)

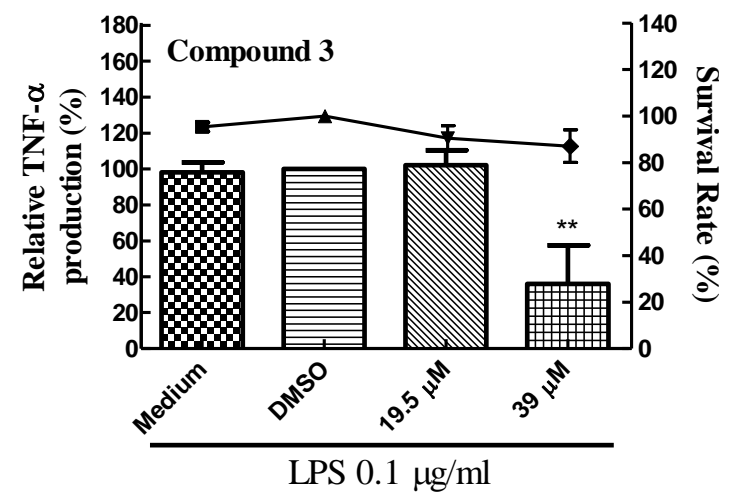

(B)

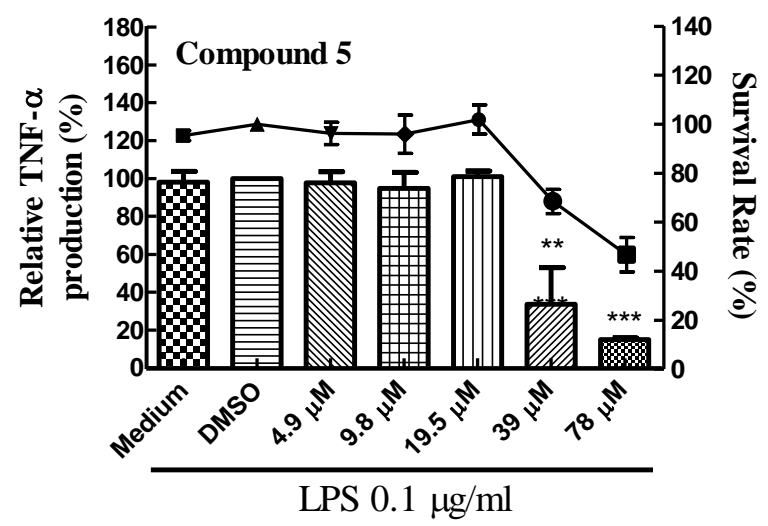

(B)

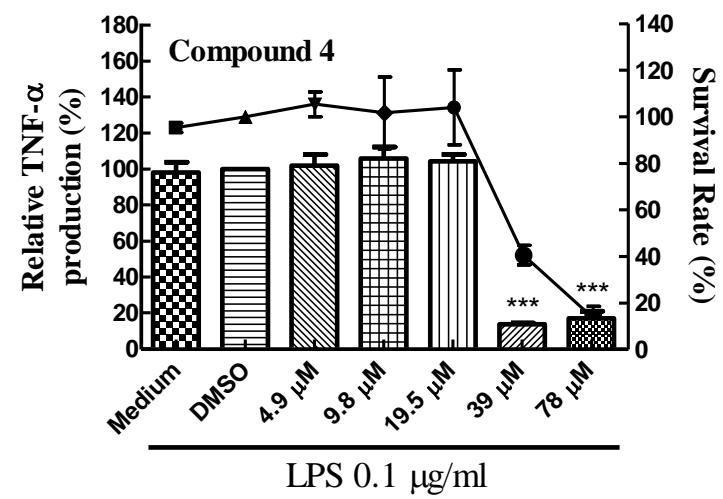

(B)

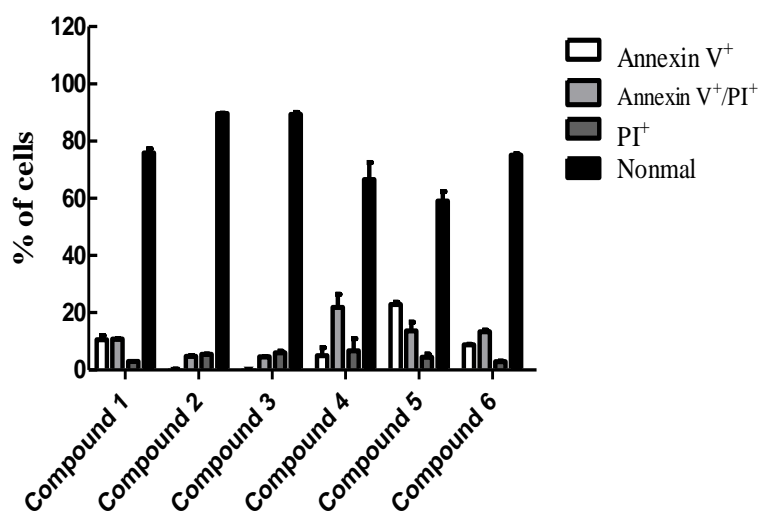

(C) 
Figure 2. Inhibitory effect of lobocrassin B on various TLR agonists-stimulating TNF- $\alpha$ production. (A) Pre-treatment of BMDCs with lobocrassin B $(39 \mu \mathrm{M})$ before LPS stimulation. (B) Post-treatment of LPS-stimulated BMDCs with lobocrassin B. (C) Inhibitory capacity of lobocrassin B on LPS-stimulated BMDCs. BMDCs were pre-treated with lobocrassin $\mathrm{B}(39 \mu \mathrm{M})$ for $1 \mathrm{~h}$ and followed by incubating with increasing dosages of LPS $(10,50,250,500$ and $1000 \mathrm{ng} / \mathrm{mL})$ for a total $6 \mathrm{~h}$. Cell culture supernatants were collected at $6 \mathrm{~h}$ after LPS stimulation, and TNF- $\alpha$ levels were measured by ELISA. The data present as the mean \pm SD. $* p<0.05$ vs. LPS-stimulated control; ** $p<0.01$ vs. LPS-stimulated control; *** $p<0.001 v s$. LPS-stimulated control. (D) Inhibitory effect of lobocrassin B on various TLR ligands-stimulating BMDCs. BMDCs were pre-treated with lobocrassin B $(39 \mu \mathrm{M})$ for $1 \mathrm{~h}$ and then stimulated with LPS (100 ng/mL), Zymosan $(20 \mu \mathrm{g} / \mathrm{mL})$, Pam $(20 \mathrm{ng} / \mathrm{mL})$ and LTA $(20 \mu \mathrm{g} / \mathrm{mL})$. Cell culture supernatants were harvested at $6 \mathrm{~h}$, and TNF- $\alpha$ levels were measured by ELISA. The data present as the mean \pm SD. $* * * p<0.001$ $v s$. individual TLR ligand-stimulated control.

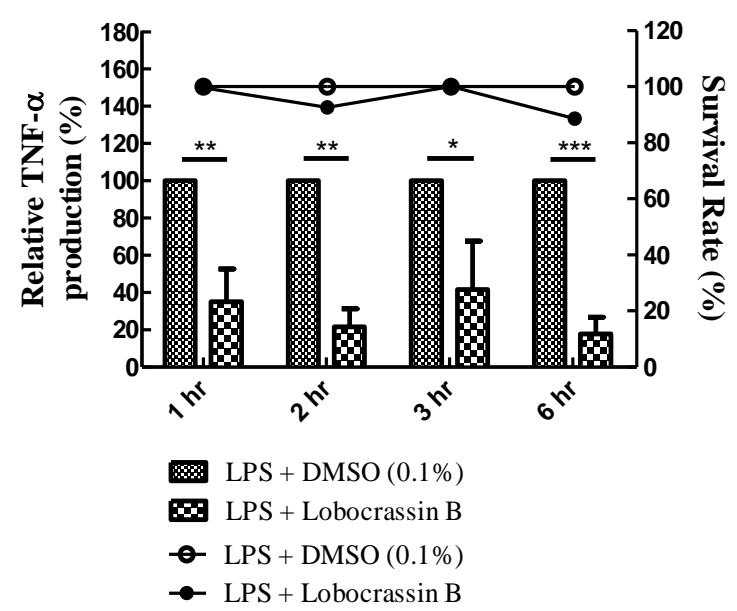

(A)

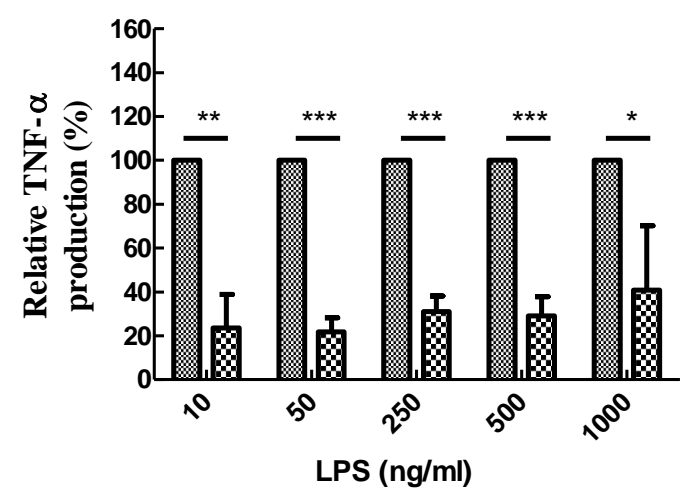

LPS $m$ LPS + Lobocrassin B

(C)

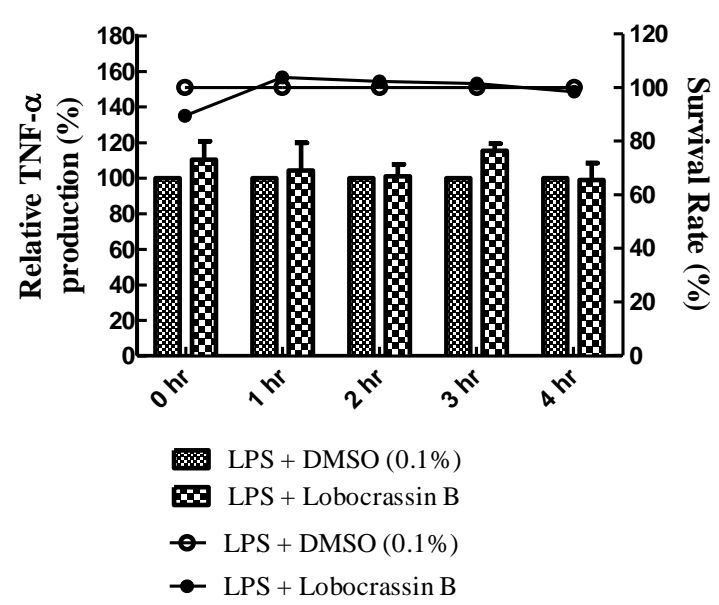

(B)

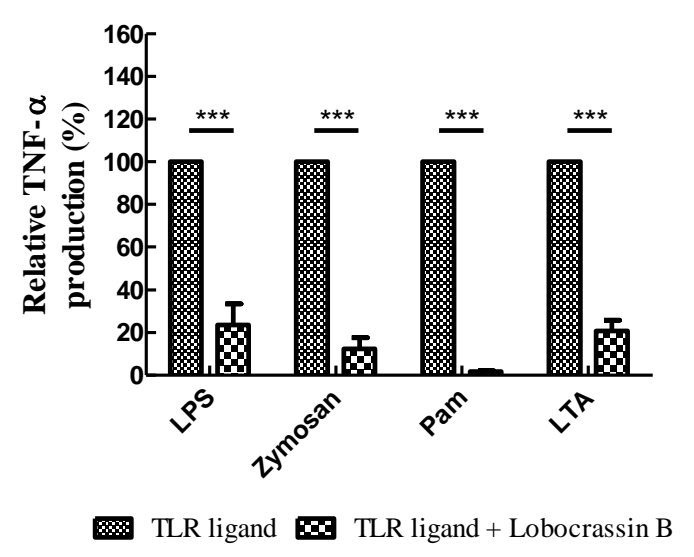

(D)

\subsection{Dose-Dependent Inhibition of TNF- $\alpha$ Production and NF- $\kappa B$ (p65) Nuclear Translocation by}

\section{Lobocrassin B}

Activation of BMDCs by TLR agonists (e.g., LPS) can transduce signaling cascade involving the downstream NF- $\kappa$ B transcription factor that may dissociate from I- $\kappa$ B in cytoplasm and then translocate 
into the nucleus to activate cytokine and chemokine genes. The production of TNF- $\alpha$ was inhibited by lobocrassin $\mathrm{B}$ in a dose-dependent manner (Figure $3 \mathrm{~A}$ ), and the corresponding LPS-mediated NF- $\mathrm{B}$ nuclear translocation was also attenuated as assayed by high content image analysis (Figure 3B,C). These results revealed that lobocrassin B indeed affects the signaling transduction mediated by TLRs.

Figure 3. Dose-dependent inhibition of TNF- $\alpha$ production and NF- $\kappa B$ (p65) nuclear translocation in LPS-stimulating BMDCs by lobocrassin B. (A) Dose-dependent inhibition of TNF- $\alpha$ production by lobocrassin B. BMDCs were pre-treated with indicated concentrations of lobocrassin B $(9.8,19.5,39,78$ and $156 \mu \mathrm{M})$ for $1 \mathrm{~h}$ and then followed by stimulation with $0.1 \mu \mathrm{g} / \mathrm{mL}$ of LPS for $5 \mathrm{~h}$. Culture supernatants were collected, and TNF- $\alpha$ production was assayed by ELISA. (B) Dose-dependent inhibition of NF- $\kappa$ B (p65) nuclear translocation by lobocrassin B. BMDCs were pre-treated with various doses of lobocrassin B as in (A) for $1 \mathrm{~h}$ and then stimulated with $0.1 \mu \mathrm{g} / \mathrm{mL}$ of LPS for $5 \mathrm{~h}$. Cells were fixed with $3.7 \%$ formaldehyde and stained with anti-NF- $\kappa \mathrm{B}$ (p65) RabMAb followed by the Alexa Fluor 488-conjugated anti-rabbit $\operatorname{IgG}(\mathrm{H}+\mathrm{L})$ antibody. Cells were further counterstained with Hochest 33258, and images were captured and analyzed. The arrow indicates the nuclear

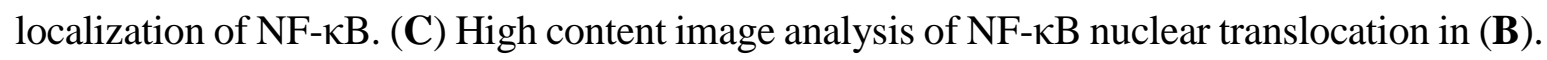
The fluorescence intensity of NF- $\mathrm{BB}$ within nucleus was analyzed by BD Attovision software. The data present as the mean \pm SD. ${ }^{*} p<0.05 v s$. LPS-stimulated control; $* * p<0.01$ vs. LPS-stimulated control; $* * * p<0.001 v s$. LPS-stimulated control.

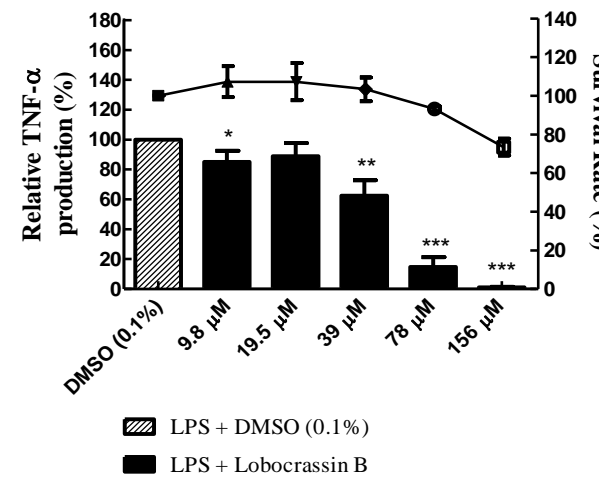

(A)

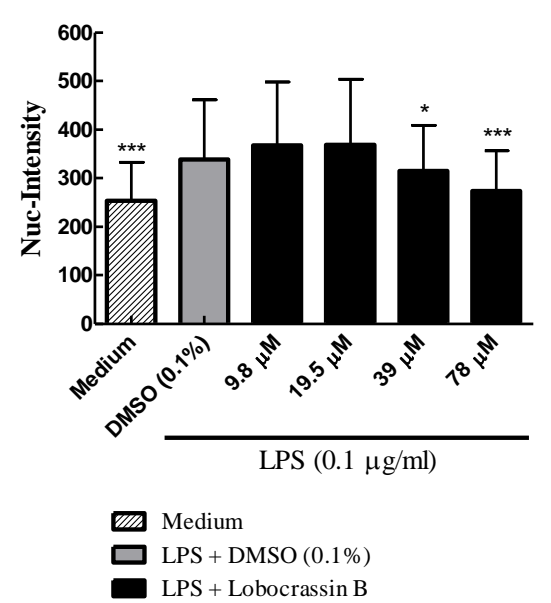

(C)
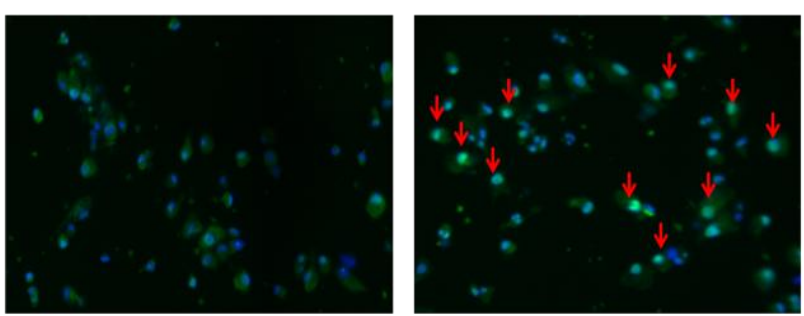

LPS DMSO $(0.1 \%)$
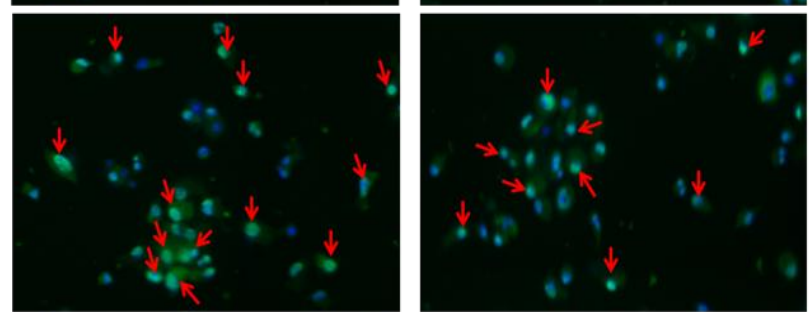

LPS Lobocrassin B $(19.5 \mu \mathrm{M})$

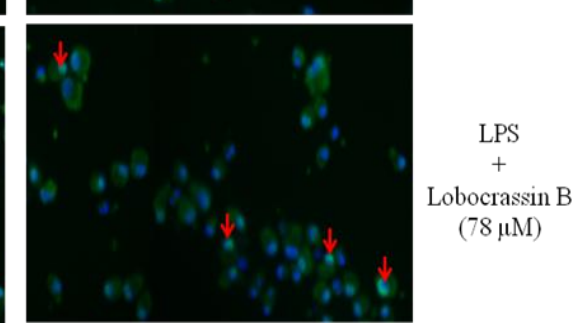

(B)

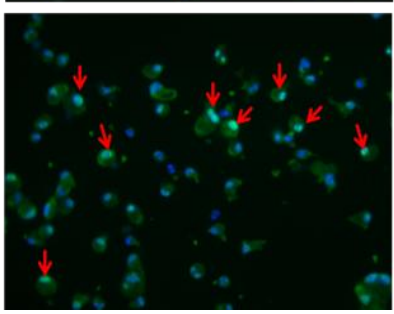

PS bocrassin $B$ $78 \mu \mathrm{M}$ 


\subsection{Attenuation of LPS-Induced DC Maturation and Endocytosis by Lobocrassin B}

DCs stimulated by invading microbes may undergo a maturation process, which in turn are capable of initiating the adequate adaptive immune responses. To investigate the effect of lobocrassin B on DC maturation, we examined the expression of MHC II and costimulatory molecules, such as CD86, CD80 and CD40 in BMDCs, which represent the key phenotypes of DC maturation. The expression levels of MHC class II, CD86, CD80 and CD40 on BMDCs were increased (mean fluorescence intensity $(\mathrm{MFI})=3.95,1.22,0.62$ and 1.76, respectively) when stimulated with LPS alone, but decreased (MFI = 1.64, 0.99, 0.49 and 1.31, respectively) as the treatment combined with LPS and lobocrassin B (Figure 4A). Additionally, we also examined if the antigen-loading ability of DCs was affected by lobocrassin B. Fluorescein isothiocyanate (FITC)-labeled dextran was incubated with BMDCs in the treatment of DMSO, lobocrassin B, LPS plus DMSO, LPS plus lobocrassin B and LPS at $4{ }^{\circ} \mathrm{C}$. Levels of endocytosis were determined by flow cytometry. LPS could enhance the endocytosis of FITC-dextran by BMDCs, but lobocrassin B alone or combined with LPS substantially reduced the uptake of FITC-dextran. The inhibitory level of endocytosis exerted by lobocrassin B was comparable to those treated at $4{ }^{\circ} \mathrm{C}$ (Figure 4B). However, the level of reactive oxygen species (ROS) within BMDCs induced by LPS were not affected by lobocrassin B, and lobocrassin B alone failed to induce ROS production (data not shown).

Figure 4. Lobocrassin B attenuated LPS-induced dendritic cell (DC) maturation and endocytosis. (A) Inhibition of BMDC maturation by lobocrassin B. BMDCs were treated with LPS $(0.1 \mu \mathrm{g} / \mathrm{mL})$ in $0.1 \%$ DMSO or LPS plus lobocrassin B $(39 \mu \mathrm{M})$ for $24 \mathrm{~h}$, and the expression levels of MHC II, CD40, CD80 and CD86 in each treatment were determined by flow cytometry. All data were gated on CD11c cells. (B) Blocking of BMDC endocytosis by lobocrassin B. BMDCs were pretreated with $0.1 \%$ DMSO or lobocrassin B $(39 \mu \mathrm{M})$ for $1 \mathrm{~h}$ and then incubated with LPS $(0.1 \mu \mathrm{g} / \mathrm{mL})$ plus dextran-FITC for an additional $1 \mathrm{~h}$. The uptake of dextran-FITC (MFI) by BMDCs was measured by flow cytometry. Negative control was conducted with BMDCs incubated with dextran-FITC at $4{ }^{\circ} \mathrm{C}$. The data present as the mean $\pm \mathrm{SD}$. $* p<0.05 v s$. DMSO control; $* * p<0.01 v s$. LPS-stimulated control.

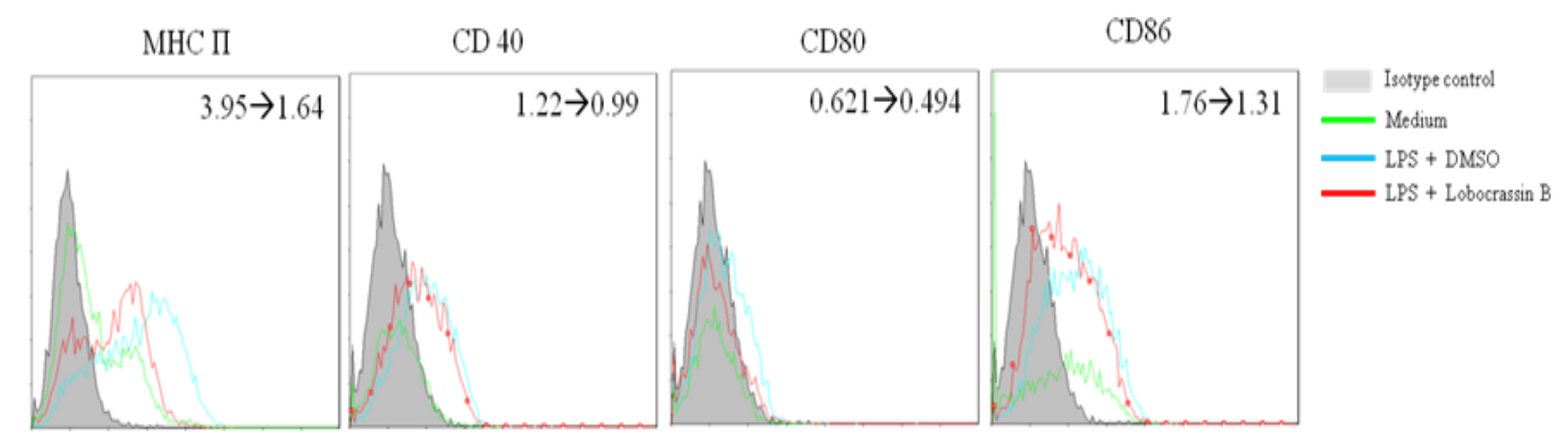

(A) 
Figure 4. Cont.

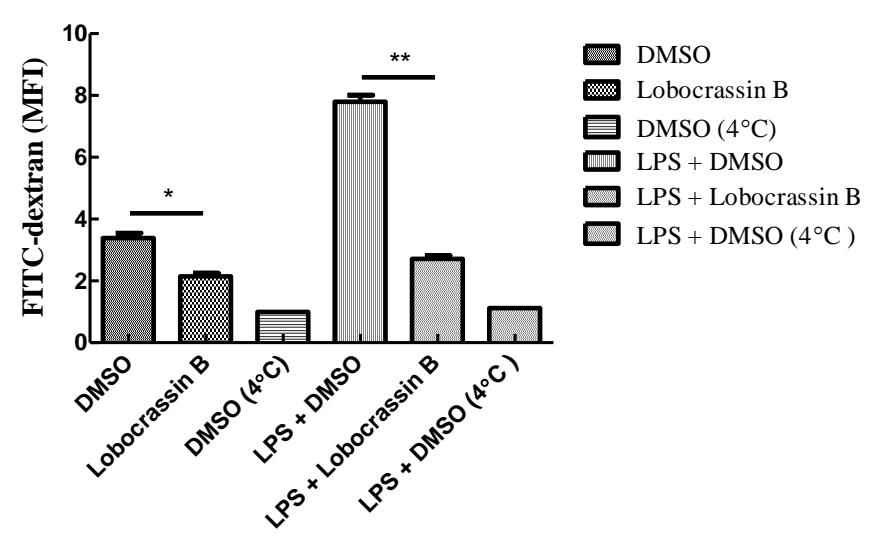

(B)

\subsection{Discussion}

Soft corals (Coelenterata, Octocorallia and Alcyonaceae) are rich in steroids and terpenoids, and mostly, the isolated diterpenes are cembrane-type compounds [22]. In previous studies, many cembranes have been found to exhibit various biological activities, such as antitumor [9,23-26], anti-microbial [27-29] and anti-inflammatory activities [10,24,25,30,31]. Within those, the cembrane-type diterpenoids with anti-inflammatory activities may decrease the expression levels of iNOS or COX-2, and somewhat upregulate TGF- $\beta$ in macrophages [13].

Here, we set up the experiments according to a previous report with minor modification, where quercetin, one of the most common flavonoids in the diet, exerted the immunosuppressive effect on dendritic cell activation and function [32]. We demonstrated that cembranolides isolated from soft coral can also suppress the activation and maturation of murine DCs. The expression levels of MHC class II, co-stimulatory molecules and adhesion molecules are lower in immature DCs, while they become high in mature DCs when inflammatory stimuli are engaged [33]. TLR receptors are engaged with the inflammatory stimuli on immature DCs and then transduce signaling to activate certain transcriptional factors (NF- $\mathrm{B}$ and AP-1), which initiate cytokine production and DC maturation [34,35]. Our results show that pre-treatment of immature DCs with cembranolides (e.g., lobocrassin B) effectively inhibits TNF- $\alpha$ production and attenuates DC maturation after LPS stimulation. Although the suppressive mechanisms mediated by lobocrassin B may not be identical to quercetin, the effective dosage of lobocrassin B used in this study is $39 \mu \mathrm{M}$, which is comparable to that $(50 \mu \mathrm{M})$ of quercetin reported previously [32].

Additionally, NF- $\mathrm{B}$ nuclear translocation was also inhibited dose-dependently by lobocrassin $\mathrm{B}$ treatment. In particular, marine natural products have recently been recognized as a promising source of NF-кB inhibitors [36-38]. The cembrane-type diterpenoids isolated from soft corals (Sarcophyton sp. and Sinularia sp.) have been shown to inhibit both TNF- $\alpha$-induced NF- $\kappa B-D N A$ binding, as well as TNF- $\alpha$-induced I- $\kappa B$ degradation and nuclear translocation of $\mathrm{p} 50 / \mathrm{p} 65$ [39]. As NF- $\kappa \mathrm{B}$ is the downstream molecule along with various TLRs-mediated signaling pathways, targeting of this molecule has a promising effect when various TLR agonists are engaged. Our results showed that lobocrassin B can antagonize the actions of various TLR ligands, such as LPS, Zymosan, Pam or LTA, by inhibiting the TNF- $\alpha$ production and the NF- $\kappa$ B activation in activated DCs. Thus, lobocrassin B may act as a NF- $\kappa \mathrm{B}$ inhibitor in accordance with the finding that cembrane-type diterpenoids may interfere in the action of 
$\mathrm{NF}-\kappa \mathrm{B}[39]$ and could reduce the expression levels of downstream iNOS and COX-2 in anti-inflammatory responses. Suppression of the ROS production induced by LPS may be one reason to decrease NF- $\mathrm{B}$ activity [40]; however, it seems not to be the action by lobocrassin B, as the induced intracellular ROS was not affected after lobocrassin B treatment (data not shown). In this study, our results suggest that cembrane-type diterpenoids isolated from the soft coral, Lobophytum crassum, may have an immunomodulatory effect on DCs and needs to be further studied in the treatment of those immune dysregulated diseases in the future.

\section{Experimental Section}

\subsection{Mice and Generation of DCs}

Male mice aged at 6-8 weeks were purchased from the National Laboratory's Animal Center (Taipei, Taiwan) and were kept in a temperature-controlled environment $\left(22{ }^{\circ} \mathrm{C}\right)$ with $70 \%$ relative humidity under a $12 \mathrm{~h}$ light/dark cycle. The animal experiments were performed according to the "Guide for the Care and Use of Laboratory Animals" of the National Dong-Hwa University. Bone marrow-derived dendritic cells (BMDCs) were generated from C57BL/6 mice bone marrow, as described previously [41,42]. Briefly, bone marrow cells were cultured in RPMI 1640 medium containing $10 \%$ fetal bovine serum, $2 \mathrm{mM}$ of L-glutamine (Gibco BRL, Grand Island, NY, USA), streptomycin-penicillin (Biowest, Nuaillé, France), 50 ng/mL of GM-CSF and IL-4 (PeproTech, Rocky Hill, NJ, USA) for seven days. Half of the culture medium was replaced by fresh complete medium every 2-3 days. On day 7 , cells were harvested and assayed for CD11c expression (a DC specific marker) by staining with PE-conjugated anti-CD11c antibody (AbD serotec, Raleigh, NC, USA). The percentage of immature DCs $\left(\mathrm{CD} 11 \mathrm{c}^{+}\right)$was determined by FC500 flow cytometer (Beckmen Coulter, Taipei, Taiwan), and immature DCs on average accounted for $70 \%$ of total bone marrow cells in each preparation.

\subsection{Preparation of Cembrane-Type Diterpenoids}

Cembrane-type diterpenoids were isolated from a soft coral, Lobophytum crassum, and the extraction procedure was described previously [5]. Their chemical structures were previously identified as (9E,13E)-5-Acetoxy-6-hydroxy-9,13-dimethyl-3-methylene-3,3a,4,5,6,7,8,11,12,14a-decahydro-2H-cy clotrideca[b]furan-2-one (1) [43], (9E,13E)-5-Acetoxy-6-acetyl-9,13-dimethyl-3-methylene-3,3a,4,5, 6,7,8,11,12,14a-decahydro-2H-cyclotrideca[b]furan-2-one (2) [43], lobocrassin B (3) [5], (-)14-deoxycrassin (4) [44], cembranolide B (5) [45] and 13-acetoxysarcocrassolide (6) [46]. Pure cembrane-type diterpenoids (1-6) were dissolved in dimethyl sulfoxide (DMSO) (Sigma-Aldrich, St. Louis, MO, USA) as stock solutions and further diluted in serum-free RPMI 1640 medium. The concentration of DMSO used in all experiments was less than $0.1 \%$.

\subsection{Cytotoxicity Assay of Cembrane-Type Diterpenoids}

Immature DCs were treated with various marine cembranolides in the absence or presence of $0.1 \mu \mathrm{g} / \mathrm{mL}$ LPS (Sigma-Aldrich) for 6 or $24 \mathrm{~h}$ and then incubated with 3-[4,5-dimethylthiazol-2-yl]-2, 5-diphenyltetrazolium bromide (MTT, concentration $2.5 \mathrm{mg} / \mathrm{mL}$ ) (Sigma-Aldrich, St. Louis, MO, USA) for $4 \mathrm{~h}$. The formazan crystal in purple color was developed from tetrazolium (MTT) within cells 
by the action of mitochondrial succinate dehydrogenase and was extracted into DMSO. The optical density (OD) of absorbance at $570 \mathrm{~nm}$ was measured by EnSpire ${ }^{\circledR}$ Multimode Plate Reader (PerkinElmer, Santa Clara, CA, USA). The survival percentage of each group was calculated by $\left(\mathrm{OD}_{570}\right.$ of treatment $/ \mathrm{OD}_{570}$ of controls) $\times 100 \%$. A direct cytotoxicity assay was conducted using an Annexin V kit (Santa Cruz Biotechnology, Dallas, TX, USA), according to the manufacturer's instructions. Apoptosis was determined by flow cytometry.

\subsection{Measurement of TNF- $\alpha$ Production}

BMDCs $\left(2 \times 10^{5} /\right.$ well in 96-well plate) were incubated with indicated marine cembranolides, as listed in Table 1, or TLR ligands, including LPS (100 ng/mL), zymosan $(20 \mu \mathrm{g} / \mathrm{mL})$, lipoteichoic acid (LTA) $(20 \mu \mathrm{g} / \mathrm{mL})$ (Sigma-Aldrich, St. Louis, MO, USA) and synthetic diacylated lipoprotein Pam2Cys-Ser-Lys4 (20 ng/mL) (InvivoGen, San Diego, CA, USA), and after $6 \mathrm{~h}$, cell culture supernatants were collected and assayed for TNF- $\alpha$ levels by mTNF- $\alpha$ kit (eBioscience), according to the manufacturer's instructions. TLR ligands were used to stimulate DCs [32]. The detection limit of $\mathrm{mTNF}-\alpha$ is $10 \mathrm{pg} / \mathrm{mL}$.

\subsection{High Content Image Analysis of NF- $\kappa B$ Nuclear Translocation}

BMDCs $\left(5 \times 10^{4} /\right.$ well in BD 96-well imaging plate) were pre-treated with lobocrassin $\mathrm{B}$ at the indicated concentration of $9.8,19.5,39$ and $78 \mu \mathrm{M}$ for $1 \mathrm{~h}$ and then stimulated with $0.1 \mu \mathrm{g} / \mathrm{mL}$ of LPS for $5 \mathrm{~h}$. The cells were fixed by cold methanol (Macron Chemicals) for $15 \mathrm{~min}$ and then washed trice using phosphate buffered saline (PBS). Cells were then stained with anti-NF- $\kappa$ B (p65) RabMAb (1:400 diluted in PBS) (Epitomics) at $4{ }^{\circ} \mathrm{C}$ overnight and washed 3 times with PBS followed by staining with $4 \mu \mathrm{g} / \mathrm{mL}$ of secondary Alexa Fluor 488 goat anti-rabbit IgG $(\mathrm{H}+\mathrm{L})$ (Invitrogen, Carlsbad, CA, USA) for 30 min. Cells were counterstained with Hochest $33258(2 \mu \mathrm{g} / \mathrm{mL})$ (Invitrogen) and subjected to image analysis using BD pathway 435 image analyzer (Becton, Dickinson and Company, BD, Franklin, NJ, USA). The fluorescence intensity of NF- $\mathrm{BB}$ (p65) within cells was analyzed by BD software, Attovision, according to the software's user manual. More than 200 cells were analyzed in each group.

\subsection{Assay of DC Maturation and Endocytosis Activity}

DC maturation was evaluated by the upregulation of MHC class II and the costimulatory molecule expression, as described previously [32]. BMDCs were treated with LPS $(0.1 \mu \mathrm{g} / \mathrm{mL})$ plus DMSO $(0.1 \%)$ or LPS plus lobocrassin B $(39 \mu \mathrm{M})$ for $24 \mathrm{~h}$. Cells were harvested and stained with mAbs specific for mouse CD11c, I-A ${ }^{\mathrm{b}}, \mathrm{CD} 40, \mathrm{CD} 80, \mathrm{CD} 86$ (AbD serotec), MHC-II (eBioscience, San Diego, CA, USA) and negative isotype control (AbD serotec and eBioscience). The mean fluorescence intensity (MFI) for each molecule was analyzed by flow cytometry. The endocytosis by BMDCs was estimated by dextran-FITC uptake, as described. BMDCs were treated with $0.1 \%$ DMSO, lobocrassin B $(39 \mu \mathrm{M})$, LPS $(0.1 \mu \mathrm{g} / \mathrm{mL})$ plus DMSO or LPS plus lobocrassin B for $1 \mathrm{~h}$ and then incubated with dextran-FITC (MW $\sim 77 \mathrm{kD}$, Sigma-Aldrich, St. Louis, MO, USA) for another $1 \mathrm{~h}$. Cells were collected and labeled with PE-conjugated anti-mouse CD11c before acquisition. The uptake of dextran-FITC by BMDCs 
$\left(\mathrm{CD} 11 \mathrm{c}^{+}\right)$was analyzed by flow cytometry. A negative control was conducted with cells incubated with dextran-FITC at $4{ }^{\circ} \mathrm{C}$.

\section{Conclusions}

Here, we examine the effects of marine cembrane-type diterpenoids, especially lobocrassin B, on mouse DCs. Lobocrassin B alone did not stimulate TNF- $\alpha$ production from BMDCs, but effectively inhibited LPS-induced DC activation by inhibiting the production of TNF- $\alpha$. Pretreatment with lobocrassin B prohibited the activation of BMDCs induced by LPS, while post-treatment exerted no obvious effects on TNF- $\alpha$ production. In addition, lobocrassin B exhibited a broad spectrum of inhibiting DC activation mediated by various TLR agonists, such as LPS (for TLR4), zymosan (for TLR2), LTA (for TLR2) and Pam2CSK4 (for TLR2). NF- $\kappa B$, an important transcription factor responsible for cytokine production in the downstream of TLR signaling, was also affected by lobocrassin B, as its nuclear translocation upon activation was inhibited. Lobocrassin B also attenuated DC maturation and endocytosis, as the expression levels of MHC class II and the costimulatory molecules, such as CD80, CD86 and CD40, were decreased in treatments, which may attenuate the function of DCs to activate naive $\mathrm{T}$-cells during the initiation of adaptive immune responses. Thus, the application of lobocrassin $\mathrm{B}$ in the treatment of immune dysregulated diseases will be further studied in the future.

\section{Acknowledgments}

We are grateful for the financial support received from the National Science Council of Taiwan (NSC 98-2320-B-259-001-MY2; NSC 100-2320-B-259-001; NSC 100-2120-M-259-002) and STSP grant EG310815101 (for C.L. Chu).

\section{References}

1. Mayer, A.M.; Rodriguez, A.D.; Berlinck, R.G.; Hamann, M.T. Marine pharmacology in 2005-6: Marine compounds with anthelmintic, antibacterial, anticoagulant, antifungal, anti-inflammatory, antimalarial, antiprotozoal, antituberculosis, and antiviral activities; affecting the cardiovascular, immune and nervous systems, and other miscellaneous mechanisms of action. Biochim. Biophys. Acta 2009, 1790, 283-308.

2. Mayer, A.M.; Rodriguez, A.D.; Berlinck, R.G.; Fusetani, N. Marine pharmacology in 2007-8: Marine compounds with antibacterial, anticoagulant, antifungal, anti-inflammatory, antimalarial, antiprotozoal, anti-tuberculosis, and antiviral activities; affecting the immune and nervous system, and other miscellaneous mechanisms of action. Comp. Biochem. Physiol. C 2011, 153, 191-222.

3. Mayer, A.M.; Rodriguez, A.D.; Berlinck, R.G.; Hamann, M.T. Marine pharmacology in 2003-4: Marine compounds with anthelmintic antibacterial, anticoagulant, antifungal, anti-inflammatory, antimalarial, antiplatelet, antiprotozoal, anti-tuberculosis, and antiviral activities; affecting the cardiovascular, immune and nervous systems, and other miscellaneous mechanisms of action. Comp. Biochem. Physiol. C 2007, 145, 553-581. 
4. Hegazy, M.E.; Su, J.H.; Sung, P.J.; Sheu, J.H. Cembranoids with 3,14-ether linkage and a secocembrane with bistetrahydrofuran from the Dongsha Atoll soft coral Lobophytum sp. Mar. Drugs 2011, 9, 1243-1253.

5. Kao, C.Y.; Su, J.H.; Lu, M.C.; Hwang, T.L.; Wang, W.H.; Chen, J.J.; Sheu, J.H.; Kuo, Y.H.; Weng, C.F.; Fang, L.S.; et al. Lobocrassins A-E: New cembrane-type diterpenoids from the soft coral Lobophytum crassum. Mar. Drugs 2011, 9, 1319-1331.

6. Lee, N.L.; Su, J.H. Tetrahydrofuran cembranoids from the cultured soft coral Lobophytum crassum. Mar. Drugs 2011, 9, 2526-2536.

7. Lin, S.T.; Wang, S.K.; Duh, C.Y. Cembranoids from the Dongsha Atoll soft coral Lobophytum crassum. Mar. Drugs 2011, 9, 2705-2716.

8. Bonnard, I.; Jhaumeer-Laulloo, S.B.; Bontemps, N.; Banaigs, B.; Aknin, M. New lobane and cembrane diterpenes from two comorian soft corals. Mar. Drugs 2010, 8, 359-372.

9. Chao, C.H.; Wen, Z.H.; Wu, Y.C.; Yeh, H.C.; Sheu, J.H. Cytotoxic and anti-inflammatory cembranoids from the soft coral Lobophytum crassum. J. Nat. Prod. 2008, 71, 1819-1824.

10. Wanzola, M.; Furuta, T.; Kohno, Y.; Fukumitsu, S.; Yasukochi, S.; Watari, K.; Tanaka, C.; Higuchi, R.; Miyamoto, T. Four new cembrane diterpenes isolated from an Okinawan soft coral Lobophytum crassum with inhibitory effects on nitric oxide production. Chem. Pharm. Bull. (Tokyo) 2010, 58, 1203-1209.

11. Quang, T.H.; Ha, T.T.; Minh, C.V.; Kiem, P.V.; Huong, H.T.; Ngan, N.T.; Nhiem, N.X.; Tung, N.H.; Tai, B.H.; Thuy, D.T.; et al. Cytotoxic and anti-inflammatory cembranoids from the Vietnamese soft coral Lobophytum laevigatum. Bioorg. Med. Chem. 2011, 19, 2625-2632.

12. Radhika, P.; Rao, P.R.; Archana, J.; Rao, N.K. Anti-inflammatory activity of a new sphingosine derivative and cembrenoid diterpene (lobohedleolide) isolated from marine soft corals of Sinularia crassa TIXIER-DURIVAULT and Lobophytum species of the Andaman and Nicobar Islands. Biol. Pharm. Bull. 2005, 28, 1311-1313.

13. Huang, S.Y.; Chen, N.F.; Chen, W.F.; Hung, H.C.; Lee, H.P.; Lin, Y.Y.; Wang, H.M.; Sung, P.J.; Sheu, J.H.; Wen, Z.H. Sinularin from indigenous soft coral attenuates nociceptive responses and spinal neuroinflammation in carrageenan-induced inflammatory rat model. Mar. Drugs 2012, 10, 1899-1919.

14. Steinman, R.M. The dendritic cell system and its role in immunogenicity. Annu. Rev. Immunol. 1991, 9, 271-296.

15. Van Vliet, S.J.; den Dunnen, J.; Gringhuis, S.I.; Geijtenbeek, T.B.; van Kooyk, Y. Innate signaling and regulation of Dendritic cell immunity. Curr. Opin. Immunol. 2007, 19, 435-440.

16. Steinman, R.M.; Inaba, K.; Turley, S.; Pierre, P.; Mellman, I. Antigen capture, processing, and presentation by dendritic cells: recent cell biological studies. Hum. Immunol. 1999, 60, 562-567.

17. Kawai, T.; Akira, S. TLR signaling. Cell Death Differ. 2006, 13, 816-825.

18. Richmond, A. NF- $\mathrm{BB}$, chemokine gene transcription and tumour growth. Nat. Rev. Immunol. 2002, 2, 664-674.

19. Steinman, R.M.; Banchereau, J. Taking dendritic cells into medicine. Nature 2007, 449, 419-426.

20. Galkina, E.; Ley, K. Immune and inflammatory mechanisms of atherosclerosis (*). Annu. Rev. Immunol. 2009, 27, 165-197. 
21. Oyoshi, M.K.; He, R.; Kumar, L.; Yoon, J.; Geha, R.S. Cellular and molecular mechanisms in atopic dermatitis. Adv. Immunol. 2009, 102, 135-226.

22. Blunt, J.W.; Copp, B.R.; Munro, M.H.; Northcote, P.T.; Prinsep, M.R. Marine natural products. Nat. Prod. Rep. 2011, 28, 196-268.

23. Su, J.H.; Ahmed, A.F.; Sung, P.J.; Chao, C.H.; Kuo, Y.H.; Sheu, J.H. Manaarenolides A-I, diterpenoids from the soft coral Sinularia manaarensis. J. Nat. Prod. 2006, 69, 1134-1139.

24. Lin, W.Y.; Su, J.H.; Lu, Y.; Wen, Z.H.; Dai, C.F.; Kuo, Y.H.; Sheu, J.H. Cytotoxic and anti-inflammatory cembranoids from the Dongsha Atoll soft coral Sarcophyton crassocaule. Bioorg. Med. Chem. 2010, 18, 1936-1941.

25. Lin, W.Y.; Lu, Y.; Su, J.H.; Wen, Z.H.; Dai, C.F.; Kuo, Y.H.; Sheu, J.H. Bioactive cembranoids from the dongsha atoll soft coral Sarcophyton crassocaule. Mar. Drugs 2011, 9, 994-1006.

26. Su, J.H.; Wen, Z.H. Bioactive cembrane-based diterpenoids from the soft coral Sinularia triangular. Mar. Drugs 2011, 9, 944-951.

27. Rashid, M.A.; Gustafson, K.R.; Boyd, M.R. HIV-Inhibitory cembrane derivatives from a Philippines collection of the soft coral Lobophytum species. J. Nat. Prod. 2000, 63, 531-533.

28. Matthee, G.F.; Konig, G.M.; Wright, A.D. Three new diterpenes from the marine soft coral Lobophytum crassum. J. Nat. Prod. 1998, 61, 237-240.

29. Tello, E.; Castellanos, L.; Arevalo-Ferro, C.; Duque, C. Cembranoid diterpenes from the Caribbean sea whip Eunicea knighti. J. Nat. Prod. 2009, 72, 1595-1602.

30. Lu, Y.; Huang, C.Y.; Lin, Y.F.; Wen, Z.H.; Su, J.H.; Kuo, Y.H.; Chiang, M.Y.; Sheu, J.H. Anti-inflammatory cembranoids from the soft corals Sinularia querciformis and Sinularia granosa. J. Nat. Prod. 2008, 71, 1754-1759.

31. Lu, Y.; Su, J.H.; Huang, C.Y.; Liu, Y.C.; Kuo, Y.H.; Wen, Z.H.; Hsu, C.H.; Sheu, J.H. Cembranoids from the soft corals Sinularia granosa and Sinularia querciformis. Chem. Pharm. Bull. (Tokyo) 2010, 58, 464-466.

32. Huang, R.Y.; Yu, Y.L.; Cheng, W.C.; OuYang, C.N.; Fu, E.; Chu, C.L. Immunosuppressive effect of quercetin on dendritic cell activation and function. J. Immunol. 2010, 184, 6815-6821.

33. Cella, M.; Engering, A.; Pinet, V.; Pieters, J.; Lanzavecchia, A. Inflammatory stimuli induce accumulation of MHC class II complexes on dendritic cells. Nature 1997, 388, 782-787.

34. Ardeshna, K.M.; Pizzey, A.R.; Devereux, S.; Khwaja, A. The PI3 kinase, p38 SAP kinase, and $\mathrm{NF}-\kappa \mathrm{B}$ signal transduction pathways are involved in the survival and maturation of lipopolysaccharide-stimulated human monocyte-derived dendritic cells. Blood 2000, 96, 1039-1046.

35. Watts, C.; West, M.A.; Zaru, R. TLR signalling regulated antigen presentation in dendritic cells. Curr. Opin. Immunol. 2010, 22, 124-130.

36. Folmer, F.; Jaspars, M.; Dicato, M.; Diederich, M. Marine natural products as targeted modulators of the transcription factor NF-кB. Biochem. Pharmacol. 2008, 75, 603-617.

37. Choi, I.K.; Shin, H.J.; Lee, H.S.; Kwon, H.J. Streptochlorin, a marine natural product, inhibits NF- $\kappa \mathrm{B}$ activation and suppresses angiogenesis in vitro. J. Microbiol. Biotechnol. 2007, 17, 1338-1343.

38. Terracciano, S.; Aquino, M.; Rodriquez, M.; Monti, M.C.; Casapullo, A.; Riccio, R.; Gomez-Paloma, L. Chemistry and biology of anti-inflammatory marine natural products: molecules interfering with cyclooxygenase, NF-кB and other unidentified targets. Curr. Med. Chem. 2006, 13, 1947-1969. 
39. Folmer, F.; Jaspars, M.; Solano, G.; Cristofanon, S.; Henry, E.; Tabudravu, J.; Black, K.; Green, D.H.; Kupper, F.C.; Aalbersberg, W.; et al. The inhibition of TNF- $\alpha$-induced NF-kB activation by marine natural products. Biochem. Pharmacol. 2009, 78, 592-606.

40. Bowie, A.; O’Neill, L.A. Oxidative stress and nuclear factor-kappaB activation: A reassessment of the evidence in the light of recent discoveries. Biochem. Pharmacol. 2000, 59, 13-23.

41. Chu, C.L.; Lowell, C.A. The Lyn tyrosine kinase differentially regulates dendritic cell generation and maturation. J. Immunol. 2005, 175, 2880-2889.

42. Lu, M.C.; Hwang, S.L.; Chang, F.R.; Chen, Y.H.; Chang, T.T.; Hung, C.S.; Wang, C.L.; Chu, Y.H.; Pan, S.H.; Wu, Y.C. Immunostimulatory effect of Antrodia camphorata extract on functional maturation of dendritic cells. Food Chem. 2009, 113, 1049-1057.

43. Yamada, Y.; Suzuki, S.; Iguchi, K.; Kikuchi, H.; Tsukitani, Y.; Horiai, H.; Shibayama, F. Studies on marine natural products. IV The stereochemistry of 13-membered carbocyclic cembranolide diterpenes from the soft coral Lobophytum pauciflorum (Ehrenberg). Tetrahedron Lett. 1980, 21, 3911-3914.

44. Wen, T.; Ding, Y.; Deng, Z.; van Ofwegen, L.; Proksch, P.; Lin, W. Sinulaflexiolides A-K, cembrane-type diterpenoids from the chinese soft coral Sinularia flexibilis. J. Nat. Prod. 2008, 71, 1133-1140.

45. Kusumi, T.; Ohtani, I.; Inouye, Y.; Kakisawa, H. Absolute configurations of cytotoxic marine cembranolides; Consideration of mosher's method. Tetrahedron Lett. 1988, 29, 4731-4734.

46. Duh, C.Y.; Wang, S.K.; Chung, S.G.; Chou, G.C.; Dai, C.F. Cytotoxic cembrenolides and steroids from the formosan soft coral Sarcophyton crassocaule. J. Nat. Prod. 2000, 63, 1634-1637.

(C) 2013 by the authors; licensee MDPI, Basel, Switzerland. This article is an open access article distributed under the terms and conditions of the Creative Commons Attribution license (http://creativecommons.org/licenses/by/3.0/). 